\title{
ORTHOGONAL POLYNOMIALS DEFINED BY A \\ RECURRENCE RELATION
}

BY

PAUL G. NEVAI ${ }^{1}$

ABSTRACT. R. Askey has conjectured that if a system of orthogonal polynomials is defined by the three term recurrence relation

$$
x p_{n-1}(x)=\frac{\gamma_{n-1}}{\gamma_{n}} p_{n}(x)+\alpha_{n-1} p_{n-1}(x)+\frac{\gamma_{n-2}}{\gamma_{n-1}} p_{n-2}(x)
$$

and

$$
\begin{aligned}
\alpha_{n} & =\frac{(-1)^{n}}{n} \text { const }+O\left(\frac{1}{n^{2}}\right), \\
\frac{\gamma_{n}}{\gamma_{n+1}} & =\frac{1}{2}+\frac{(-1)^{n}}{n} \text { const }+O\left(\frac{1}{n^{2}}\right),
\end{aligned}
$$

then the logarithm of the absolutely continuous portion of the corresponding weight function is integrable. The purpose of this paper is to prove $\mathbf{R}$. Askey's conjecture and solve related problems.

Let $\alpha$ be a nondecreasing function defined on the real line. Such a function $\alpha$ is called weight function if it takes infinitely many values and all its moments are finite. ${ }^{2}$ For a given weight $\alpha$ there exists a unique system of polynomials $\left\{p_{n}(d \alpha)\right\}_{n=0}^{\infty}$ such that $p_{n}(d \alpha, x)=\gamma_{n}(d \alpha) x^{n}+\ldots\left(\gamma_{n}>0\right)$ and

$$
\int_{-\infty}^{\infty} p_{n} p_{m} d \alpha=\delta_{n m}
$$

These orthogonal polynomials satisfy the recurrence formula

$$
\begin{aligned}
x p_{n-1}(d \alpha, x)= & \frac{\gamma_{n-1}(d \alpha)}{\gamma_{n}(d \alpha)} p_{n}(d \alpha, x)+\alpha_{n-1}(d \alpha) p_{n-1}(d \alpha, x) \\
& +\frac{\gamma_{n-2}(d \alpha)}{\gamma_{n-1}(d \alpha)} p_{n-2}(d \alpha, x)
\end{aligned}
$$

for $n=1,2, \ldots$ where $p_{0}=\gamma_{0}, p_{-1}=0$ and

$$
\alpha_{n}(d \alpha)=\int_{-\infty}^{\infty} x p_{n}^{2}(d \alpha, x) d \alpha(x)
$$

Received by the editors January 16, 1978 and, in revised form, July 7, 1978.

AMS (MOS) subject classifications (1970). Primary 42 A52.

'Research sponsored by the National Science Foundation under grant No. MCS75-06687.

${ }^{2}$ Many authors reserve the term "weight" for absolutely continuous $\alpha$ 's. The present paper deviates from the norm in this regard. 
It is well known that if $\operatorname{supp}(d \alpha)$ is compact then

$$
\sup _{n>0}\left|\alpha_{n}(d \alpha)\right|<\infty, \quad \sup _{n>1} \frac{\gamma_{n-1}(d \alpha)}{\gamma_{n}(d \alpha)}<\infty .
$$

On the other hand, if we take two sequences of real numbers $\left\{\alpha_{n}\right\}_{n=0}^{\infty}$ and $\left\{\gamma_{n}>0\right\}_{n=0}^{\infty}$ such that

$$
\sup _{n>0}\left|\alpha_{n}\right|<\infty, \quad \sup _{n>1} \frac{\gamma_{n-1}}{\gamma_{n}}<\infty
$$

and we build up a system of polynomials $\left\{p_{n}\right\}_{n=0}^{\infty}$ defined by

$$
x p_{n-1}(x)=\frac{\gamma_{n-1}}{\gamma_{n}} p_{n}(x)+\alpha_{n-1} p_{n-1}(x)+\frac{\gamma_{n-2}}{\gamma_{n-1}} p_{n-2}(x),
$$

$n=1,2, \ldots, p_{0}=\gamma_{0}$ and $p_{-1}=0$ then these polynomials are orthogonal with respect to some uniquely determined weight $\alpha$ having compact support [2]. Therefore every information concerning $\alpha$ and $p_{n}$ is contained in the recurrence relation (1) whenever (2) is satisfied. It is very likely that one of the main tasks of the theory of orthogonal polynomials in the near future will be to squeeze out that information from the recurrence formula. At the present time very little is known about solutions of second order linear difference equations. There is a special case, however, when something can be said about polynomials satisfying (3). In [3], [4] and [5] a number of results were proved concerning the class $M$ which is defined by

$$
M=\left\{\alpha: \lim _{n \rightarrow \infty} \alpha_{n}(d \alpha)=0, \lim _{n \rightarrow \infty} \frac{\gamma_{n-1}(d \alpha)}{\gamma_{n}(d \alpha)}=\frac{1}{2}\right\} .
$$

Let us mention that $M$ contains many weight functions. In particular, the Szegö class $S$ is contained in $M$. Here

$$
S=\left\{\alpha: \operatorname{supp}(d \alpha)=[-1,1], \log \alpha^{\prime}(\cos \theta) \in L^{1}\right\} .
$$

Another example is the Pollaczek weight which belongs to $M \backslash S$. (See e.g. [3], [6].) Also, from $\alpha \in M$ does not follow that the support of $d \alpha$ is contained in $[-1,1]$. If, for instance, a jump is added to the Chebyshev weight then the new weight still belongs to $M$. Actually, $M \backslash S$ must be large since from $\alpha \in S$ the convergence of series

$$
\sum_{k=0}^{\infty} \alpha_{k}^{2}+\left[2 \frac{\gamma_{k}}{\gamma_{k+1}}-1\right]^{2}
$$

and

$$
\sum_{k=0}^{\infty} \alpha_{k}, \quad \sum_{k=0}^{\infty}\left[2 \frac{\gamma_{k}}{\gamma_{k+1}}-1\right]
$$

follows. (See [3].) This suggests that investigation of the class $M_{1}$ containing 
those weights $\alpha$ for which

$$
\sum_{k=0}^{\infty}\left[\left|\alpha_{k}(d \alpha)\right|+\left|2 \frac{\gamma_{k}(d \alpha)}{\gamma_{k+1}(d \alpha)}-1\right|\right]<\infty
$$

is a good starting point. It was shown in [3] that if $\alpha \in M_{1}$ then one can get asymptotic formulas for the corresponding orthogonal polynomials. It turns out, however, that $M_{1}$ is relatively small. If $\alpha \in M_{1}$ then $\alpha$ must be absolutely continuous on $(-1,1)$ and $\alpha^{\prime}$ is positive and continuous on $(-1,1)$. Despite of this it is not clear whether $\alpha \in M_{1}$ implies $\log \alpha^{\prime}(\cos \theta) \in L_{1}$. K. M. Case [1] suggested that

$$
\alpha_{k}(d \alpha)=O\left(\frac{1}{k^{2}}\right), \quad \frac{\gamma_{k}(d \alpha)}{\gamma_{k+1}(d \alpha)}=\frac{1}{2}+O\left(\frac{1}{k^{2}}\right)
$$

$(k=1,2, \ldots)$ should imply the integrability of $\log \alpha^{\prime}(\cos \theta)$. In [4] we proved that $\alpha^{\prime}$ is greater than a Jacobi weight whenever (4) is satisfied. Therefore K. M. Case was right. In this work we will show that $\log \alpha^{\prime}(\cos \theta)$ is integrable provided that series

$$
\sum_{k=3}^{\infty} \log k\left[\left|\alpha_{k}(d \alpha)\right|+\left|2 \frac{\gamma_{k}(d \alpha)}{\gamma_{k+1}(d \alpha)}-1\right|\right]
$$

converges. However, the true purpose of this paper is to solve some problems posed by R. Askey.

Let $\beta$ be defined by $\operatorname{supp}(d \beta)=[-1,1]$ and

$$
d \beta(x)=|x|^{a}\left(1-x^{2}\right)^{b} d x \quad(-1<x<1)
$$

where $a>-1$ and $b>-1$. It is clear that $\alpha_{n}(d \beta)=0$ for every $n$. R. Askey noticed that $\gamma_{n}(d \beta)$ satisfy the condition

$$
\frac{\gamma_{n}(d \beta)}{\gamma_{n+1}(d \beta)}=\frac{1}{2}+\frac{(-1)^{n} B}{n}+O\left(\frac{1}{n^{2}}\right) \quad(n=1,2, \ldots)
$$

where $B$ is some fixed real number. This led R. Askey to the conjecture that $\log \alpha^{\prime}(\cos \theta)$ should be integrable whenever

$$
\alpha_{n}(d \alpha)=\frac{(-1)^{n} D}{n}+O\left(\frac{1}{n^{2}}\right)
$$

and

$$
\frac{\gamma_{n}(d \alpha)}{\gamma_{n+1}(d \alpha)}=\frac{1}{2}+\frac{(-1)^{n} E}{n}+O\left(\frac{1}{n^{2}}\right)
$$

$(n=1,2, \ldots)$ is satisfied with some fixed numbers $D$ and $E$. Let us note that (6) and (7) are stronger than the condition 


$$
\begin{gathered}
\sum_{j=1}^{n} j\left\{\left|\alpha_{j}(d \alpha)+\alpha_{j+1}(d \alpha)\right|+\left|1-4 \frac{\gamma_{j}(d \alpha)}{\gamma_{j+2}(d \alpha)}\right|\right. \\
\left.+\alpha_{j}^{2}(d \alpha)+\left[1-2 \frac{\gamma_{j}(d \alpha)}{\gamma_{j+1}(d \alpha)}\right]^{2}\right\}<\text { const } \log n
\end{gathered}
$$

for $n=3,4, \ldots$ We will show that from (8) follows that $d \alpha$ is greater than a $d \beta$ defined by (5). Hence $\log \alpha^{\prime}(\cos \theta)$ is far from being nonintegrable if (6) and (7) hold. R. Askey had felt in the same way since he also conjectured the following. Let

$$
\alpha_{n}(d \alpha)=\frac{(-1)^{n} D}{n}+O\left(\frac{1}{n^{p}}\right)
$$

and

$$
\frac{\gamma_{n}(d \alpha)}{\gamma_{n+1}(d \alpha)}=\frac{1}{2}+\frac{(-1)^{n} E}{n}+O\left(\frac{1}{n^{p}}\right)
$$

for some $p>1$. Then $\log \alpha^{\prime}(\cos \theta)$ is integrable. We will prove that $R$. Askey was again right. Actually, $\log \alpha^{\prime}(\cos \theta)$ is integrable whenever

$$
\begin{array}{r}
\sum_{j=3}^{\infty} \log j\left\{\left|\alpha_{j}(d \alpha)+\alpha_{j+1}(d \alpha)\right|+\left|1-4 \frac{\gamma_{j}(d \alpha)}{\gamma_{j+2}(d \alpha)}\right|\right. \\
\left.+\alpha_{j}^{2}(d \alpha)+\left[1-2 \frac{\gamma_{j}(d \alpha)}{\gamma_{j+1}(d \alpha)}\right]^{2}\right\}<\infty
\end{array}
$$

We will also investigate orthogonal polynomials corresponding to weights $\alpha$ satisfying

$$
\begin{aligned}
& \sum_{j=0}^{\infty}\left\{\left|\alpha_{j}(d \alpha)+\alpha_{j+1}(d \alpha)\right|+\left|1-4 \frac{\gamma_{j}(d \alpha)}{\gamma_{j+2}(d \alpha)}\right|\right. \\
& \left.+\alpha_{j}^{2}(d \alpha)+\left[1-2 \frac{\gamma_{j}(d \alpha)}{\gamma_{j+1}(d \alpha)}\right]^{2}\right\}<\infty
\end{aligned}
$$

Let us note that if $\alpha \in M_{1}$ then (10) holds.

Before going into more details let us mention a third conjecture of $\mathbf{R}$. Askey which we cannot solve at the present time: if 


$$
\alpha_{n}(d \alpha)=\frac{D}{n}+O\left(\frac{1}{n^{2}}\right)
$$

and

$$
\frac{\gamma_{n}(d \alpha)}{\gamma_{n+1}(d \alpha)}=\frac{E}{n}+O\left(\frac{1}{n^{2}}\right)
$$

with $D^{2}+E^{2}>0$ then $\log \alpha^{\prime}(\cos \theta)$ is not integrable.

In the following by constants we mean positive numbers which are independent of $x$ and $n$. The symbol $\Delta$ will always denote a closed interval. The measure of $\Delta$ is $|\Delta|$. If $x \in[-1,1]$ then $x=\cos \theta$ with some $\theta \in[0, \pi]$. This correspondence between $x$ and $\theta$ is taken for granted. Therefore a statement like $f(\theta)$ is continuous on $\Delta \subset[-1,1]$ means that $f(\theta)$ is continuous for $\cos \theta=x \in \Delta$. We will always assume that all weights considered belong to $M$. The only exception is Lemma 1 .

LEMMA 1. Let $\operatorname{supp}(d \alpha)$ be compact. Then

$$
\begin{gathered}
\left|p_{n}(d \alpha, x)-2 \cos 2 \theta p_{n-2}(d \alpha, x)+p_{n-4}(d \alpha, x)\right| \\
\leqslant \delta_{n}\left[\left|p_{n-3}(d \alpha, x)\right|+\left|p_{n-4}(d \alpha, x)\right|\right]
\end{gathered}
$$

and

$$
\begin{gathered}
\left|p_{n}(d \alpha, x)-2 \cos 2 \theta p_{n-2}(d \alpha, x)+p_{n-4}(d \alpha, x)\right| \\
\leqslant \delta_{n}\left[\left|p_{n-2}(d \alpha, x)\right|+\left|p_{n-3}(d \alpha, x)\right|\right]
\end{gathered}
$$

for $n=4,5, \ldots$ and $|x| \leqslant 1$ where $x=\cos \theta$ and

$$
\begin{array}{r}
\delta_{n} \leqslant K \sum_{j=n-4}^{n-2}\left\{\left|\alpha_{j}(d \alpha)+\alpha_{j+1}(d \alpha)\right|+\left|1-4 \frac{\gamma_{j}(d \alpha)}{\gamma_{j+2}(d \alpha)}\right|\right. \\
\left.+\alpha_{j}^{2}(d \alpha)+\left[1-2 \frac{\gamma_{j}(d \alpha)}{\gamma_{j+1}(d \alpha)}\right]^{2}\right\}
\end{array}
$$

with $K$ depending only on the smallest interval containing $\operatorname{supp}(d \alpha)$ and $\sup _{k>0}\left\{\gamma_{k+1}(d \alpha) / \gamma_{k}(d \alpha)\right\}$.

Proof. Using repeatedly the recurrence relation we can expand $x^{2} p_{n-2}$ into Fourier series in $\left\{p_{j}\right\}$. We have 


$$
\begin{aligned}
x^{2} p_{n-2}= & \frac{\gamma_{n-2}}{\gamma_{n}} p_{n}+\frac{\gamma_{n-2}}{\gamma_{n-1}}\left[\alpha_{n-1}+\alpha_{n-2}\right] p_{n-1} \\
& +\left[\frac{\gamma_{n-2}^{2}}{\gamma_{n-1}^{2}}+\frac{\gamma_{n-3}^{2}}{\gamma_{n-2}^{2}}+\alpha_{n-2}^{2}\right] p_{n-2} \\
& +\frac{\gamma_{n-3}}{\gamma_{n-2}}\left[\alpha_{n-2}+\alpha_{n-3}\right] p_{n-3}+\frac{\gamma_{n-4}}{\gamma_{n-2}} p_{n-4}
\end{aligned}
$$

Therefore

$$
\begin{aligned}
p_{n}-2(2 & \left.x^{2}-1\right) p_{n-2}+p_{n-4} \\
= & {\left[1-4 \frac{\gamma_{n-2}}{\gamma_{n}}\right] p_{n}-4 \frac{\gamma_{n-2}}{\gamma_{n-1}}\left[\alpha_{n-1}+\alpha_{n-2}\right] p_{n-1} } \\
& -4\left[\left(\frac{\gamma_{n-2}}{\gamma_{n-1}}-\frac{\gamma_{n-3}}{\gamma_{n-2}}\right)^{2}+\frac{1}{2}\left(4 \frac{\gamma_{n-3}}{\gamma_{n-1}}-1\right)+\alpha_{n-2}^{2}\right] p_{n-2} \\
& -4 \frac{\gamma_{n-3}}{\gamma_{n-2}}\left[\alpha_{n-2}+\alpha_{n-3}\right] p_{n-3}+\left[1-4 \frac{\gamma_{n-4}}{\gamma_{n-2}}\right] p_{n-4} .
\end{aligned}
$$

The first part of the lemma will be proved if we show that

$$
\left|p_{k}(x)\right|<\operatorname{const}\left[\left|p_{n-3}(x)\right|+\left|p_{n-4}(x)\right|\right]
$$

for $k=n-2, n-1, n$ when $n=4,5, \ldots$ and $-1<x<1$. Rewriting the recurrence formulas as

$$
p_{n-2}(x)=\frac{\gamma_{n-2}}{\gamma_{n-3}}\left[\left(x-\alpha_{n-3}\right) p_{n-3}(x)-\frac{\gamma_{n-4}}{\gamma_{n-3}} p_{n-4}(x)\right]
$$

we see that for $k=n-2$ (11) holds with a constant depending on $\Delta \supset$ $\operatorname{supp}(d \alpha)$ and $\sup _{k \geq 0}\left\{\gamma_{k+1} / \gamma_{k}\right\}$. Using induction we obtain (11) also for $k=n-1$ and $k=n$. The second part of the lemma follows from

$$
\left|p_{k}(x)\right| \leqslant \text { const }\left[\left|p_{n-2}(x)\right|+\left|p_{n-3}(x)\right|\right]
$$

( $k=n-4, n-1, n ; n=4,5, \ldots$ and $-1<x<1$ ) which can be proved in the same way as (11).

LEMMA 2. If $T_{n}$ is a trigonometric polynomial of degree at most $n$ then from

$$
\max _{0<t<2 \pi}\left|T_{n}(t) \sin 2 t\right|<1
$$

the inequality

$$
\max _{0<t<2 \pi}\left|T_{n}(t)\right|<72(n+1)
$$

follows. 
Proof. Let $D_{n}(t)=\sum_{k=-n}^{n} e^{i k t}$ be the $n$th Dirichlet kernel. Then $\left|D_{n}(t)\right|<$ $D_{n}(0)=2 n+1$ and

$$
\frac{1}{2 \pi} \int_{0}^{2 \pi} D_{n}^{2}(t) d t=2 n+1
$$

Since $T_{n}$ convolved with $D_{n}$ equals $T_{n}$ the inequality

$$
\left|T_{n}(t)\right|<\frac{2 n+1}{2 \pi} \int_{0}^{2 \pi}\left|T_{n}(\theta)\right| d \theta
$$

holds for every $t$. Therefore

$$
\int_{E}\left|T_{n}(\theta)\right| d \theta<|E| \frac{2 n+1}{2 \pi} \int_{0}^{2 \pi}\left|T_{n}(\theta)\right| d \theta
$$

for every measurable set $E$, that is

$$
\int_{0}^{2 \pi}\left|T_{n}(\theta)\right| d \theta \leqslant \int_{[0,2 \pi] \backslash E}\left|T_{n}(\theta)\right| d \theta+|E| \frac{2 n+1}{2 \pi} \int_{0}^{2 \pi}\left|T_{n}(\theta)\right| d \theta .
$$

Thus we obtain that

$$
\int_{0}^{2 \pi}\left|T_{n}(\theta)\right| d \theta<2 \int_{[0,2 \pi] \backslash E} \mid T_{n}(\theta) d \theta
$$

whenever the measure of $E$ is less than $\pi /(2 n+1)$. Using (13) we get

$$
\left|T_{n}(t)\right|<\frac{2 n+1}{\pi} \int_{[0,2 \pi] \backslash E}\left|T_{n}(\theta)\right| d \theta
$$

if $0 \leqslant t \leqslant 2 \pi$ and $|E| \leqslant \pi /(2 n+1)$. Now let us replace here $T_{n}(\theta)$ by $T_{n}(\theta) D_{n}^{2}(t-\theta)$. Since the latter is a trigonometric polynomial of degree at most $3 n$ we have

$$
\left|D_{n}^{2}(0) T_{n}(t)\right|<\frac{6 n+1}{\pi} \int_{[0,2 \pi] \backslash E}\left|T_{n}(\theta)\right| D_{n}^{2}(t-\theta) d \theta
$$

for $0<t<2 \pi$ and $|E|<\pi /(6 n+1)$. Consequently by (12)

$$
\max _{0<t<2 \pi}\left|T_{n}(t)\right|<6 \sup _{t \in[0,2 \pi] \backslash E}\left|T_{n}(t)\right|
$$

provided that $|E|<\pi /(6 n+1)$. Let us choose $E$ to be

$$
\bigcup_{k=0}^{3}\left[\frac{k \pi}{2}-\frac{\pi}{8(6 n+1)}, \frac{k \pi}{2}+\frac{\pi}{8(6 n+1)}\right] \text {. }
$$

Then $|E|=\pi /(6 n+1)$ and

$$
|\sin 2 t|>\frac{2}{\pi} \cdot \frac{2 \pi}{8(6 n+1)}=\frac{1}{2(6 n+1)}
$$

for $t \in[0,2 \pi] \backslash E$. Thus

$$
\max _{0<t<2 \pi}\left|T_{n}(t)\right|<12(6 n+1) \sup _{t \in[0,2 \pi] \backslash E}\left|T_{n}(t) \sin 2 t\right|
$$

which proves the lemma. 
LEMMA 3. Let $\psi_{n}(d \alpha)$ be defined by

$$
\psi_{n}(d \alpha, \theta)=p_{n}(d \alpha, x)-e^{2 i \theta} p_{n-2}(d \alpha, x),
$$

$x=\cos \theta$ and let $\delta_{j}$ be the numbers introduced in Lemma 1 . Then for $-1<x$ $<1$ the inequalities

$$
\begin{aligned}
&\left|\psi_{n}(d \alpha, \theta)\right|+\left|\psi_{n-1}(d \alpha, \theta)\right| \\
&< {\left[\left|\psi_{n-2}(d \alpha, \theta)\right|+\left|\psi_{n-3}(d \alpha, \theta)\right|\right]\left[1+\frac{\delta_{n}+\delta_{n-1}}{|\sin 2 \theta|}\right], } \\
&\left|\psi_{n-2}(d \alpha, \theta)\right|+\left|\psi_{n-3}(d \alpha, \theta)\right| \\
&< {\left[\left|\psi_{n}(d \alpha, \theta)\right|+\left|\psi_{n-1}(d \alpha, \theta)\right|\right]\left[1+\frac{\delta_{n}+\delta_{n-1}}{|\sin 2 \theta|}\right], } \\
&\left|\psi_{n}(d \alpha, \theta)\right|<C_{1} \exp \left\{\frac{1}{|\sin 2 \theta|} \sum_{j=4}^{n} \delta_{j}\right\}
\end{aligned}
$$

and

$$
\left|\psi_{n}(d \alpha, \theta)\right|<C_{2} \exp \left\{C_{3} \sum_{j=4}^{n} j \delta_{j}\right\}
$$

hold for $n=10,11, \ldots$ where the constants $C_{1}, C_{2}$ and $C_{3}$ do not depend on $n$ and $x$.

Proof. Since

$$
\psi_{n}-e^{-2 i \theta} \psi_{n-2}=p_{n}-2 \cos 2 \theta p_{n-2}+p_{n-4}
$$

we have by Lemma 1

$$
\left|\psi_{n}-e^{-2 i \theta} \psi_{n-2}\right|<\delta_{n}\left[\left|p_{n-3}\right|+\left|p_{n-4}\right|\right]
$$

that is

$$
\left|\psi_{n}-e^{-2 i \theta_{n-2}}\right|<\delta_{n} \frac{\left|\operatorname{Im}\left(e^{-2 i \theta} \psi_{n-3}\right)\right|+\left|\operatorname{Im} \psi_{n-2}\right|}{|\sin 2 \theta|}
$$

Similarly

$$
\left|\psi_{n-1}-e^{-2 i \theta} \psi_{n-3}\right|<\delta_{n-1} \frac{\left|\operatorname{Im} \psi_{n-2}\right|+\left|\operatorname{Im} \psi_{n-3}\right|}{|\sin 2 \theta|}
$$

Therefore

$$
\left|\psi_{n}\right|<\left|\psi_{n-2}\right|+\delta_{n} \frac{\left|\psi_{n-3}\right|+\left|\psi_{n-2}\right|}{|\sin 2 \theta|}
$$

and

$$
\left|\psi_{n-1}\right|<\left|\psi_{n-3}\right|+\delta_{n-1} \frac{\left|\psi_{n-2}\right|+\left|\psi_{n-3}\right|}{|\sin 2 \theta|}
$$


Adding the last two inequalities together we obtain (15). Inequality (16) can be proved in the same way. Repeated application of (15) yields

$$
\left|\psi_{n}\right|+\left|\psi_{n-1}\right|<C_{1} \exp \left\{\frac{1}{|\sin 2 \theta|} \sum_{j=4}^{n} \delta_{j}\right\}
$$

which implies (17). To prove (18) we use Lemma 2. We have by (19) and (20)

$$
\left|\left(\psi_{n}-e^{-2 i \theta_{n-2}}\right) \sin 2 \theta\right| \leqslant \delta_{n}\left[\max \left|\psi_{n-3}\right|+\max \left|\psi_{n-2}\right|\right]
$$

and

$$
\left|\left(\psi_{n-1}-e^{-2 i \theta_{n-3}}\right) \sin 2 \theta\right| \leqslant \delta_{n-1}\left[\max \left|\psi_{n-3}\right|+\max \left|\psi_{n-2}\right|\right] .
$$

Consequently by Lemma 2

$$
\max \left|\psi_{n}-e^{-2 i \theta_{n-2}}\right| \leqslant 72(n+1) \delta_{n}\left[\max \left|\psi_{n-3}\right|+\max \left|\psi_{n-2}\right|\right]
$$

and

$$
\max \left|\psi_{n-1}-e^{-2 i \theta_{n-3}}\right| \leqslant 72 n \delta_{n-1}\left[\max \left|\psi_{n-3}\right|+\max \left|\psi_{n-2}\right|\right] .
$$

From the last two inequalities we obtain

$$
\begin{aligned}
\max \left|\psi_{n}\right| & +\max \left|\psi_{n-1}\right| \\
& \leqslant\left[\max \left|\psi_{n-2}\right|+\max \left|\psi_{n-3}\right|\right]\left[1+72(n+1) \delta_{n}+72 n \delta_{n-1}\right] .
\end{aligned}
$$

Applying repeatedly this inequality we get (18).

LEMMA 4. Let (10) be satisfied and let $\psi_{n}(d \alpha)$ be defined by (14). Then

$$
\psi(d \alpha, \theta)=\lim _{n \rightarrow \infty} e^{i n \theta} \psi_{n}(d \alpha, \theta)
$$

exists for $x \in A=(-1,1) \backslash 0$ and the convergence is uniform inside $A$. The limit function $\psi$ is continuous on $A$ and its absolute value satisfies the inequality

$$
|\psi(d \alpha, \theta)|^{ \pm 1} \leqslant \exp \{\text { const } /|\sin 2 \theta|\}
$$

for $x \in A$. Furthermore

$$
|\psi(d \alpha, \theta)|=2 \sqrt{\frac{2}{\pi}} \frac{|x|\left(1-x^{2}\right)^{1 / 4}}{\sqrt{\alpha^{\prime}(x)}}
$$

for $x \in A$. In particular, $\alpha^{\prime}$ is continuous and positive for $x \in A$.

Proof. It follows from (10) that

$$
\delta=\sum_{j=4}^{\infty} \delta_{j}<\infty
$$

Therefore by (17)

$$
\left|\psi_{n}(\theta)\right| \leqslant C_{1} \exp \{\delta /|\sin 2 \theta|\}
$$

for $n=10,11, \ldots$ and $-1<x<1(x=\cos \theta)$. Consequently the 
inequality

$$
\left|p_{n}(x)\right| \leqslant \frac{C_{1}}{|\sin 2 \theta|} \exp \left\{\frac{\delta}{|\sin 2 \theta|}\right\}
$$

$(n=10,11, \ldots$ and $-1 \leqslant x \leqslant 1)$ also holds since $\sin 2 \theta p_{n}(x)=$ $\operatorname{Im}\left\{e^{-2 i \theta} \psi_{n}(\theta)\right\}$. We have

$$
e^{2 i n \theta} \psi_{2 n}(\theta)=\sum_{k=6}^{n} e^{2 i k \theta}\left[\psi_{2 k}(\theta)-e^{-2 i \theta} \psi_{2 k-2}(\theta)\right]+e^{10 i \theta} \psi_{10}(\theta) .
$$

By Lemma 1 and (24) the series

$$
\sum_{k=6}^{\infty} e^{2 i k \theta}\left[\psi_{2 k}(\theta)-e^{-2 i \theta} \psi_{2 k-2}(\theta)\right]
$$

converges uniformly inside $A=(-1,1) \backslash 0$. Thus

$$
\psi(\theta)=\lim _{n \rightarrow \infty} e^{2 i n \theta} \psi_{2 n}(\theta)
$$

exists and it is continuous for $x \in A$. By (23)

$$
|\psi(\theta)|<C_{1} \exp \{\delta /|\sin 2 \theta|\} .
$$

The next step is to show that $e^{(2 n+1) i \theta} \psi_{2 n+1}(\theta)$ converges to the same function $\psi(\theta)$ when $n \rightarrow \infty$ and again the convergence is uniform inside $A$. By the recurrence formula

$$
\begin{aligned}
2 x \psi_{2 n+1} & (\theta)-\psi_{2 n}(\theta)-\psi_{2 n+2}(\theta) \\
= & 2 x p_{2 n+1}(x)-p_{2 n}(x)-p_{2 n+2}(x) \\
& -e^{2 i \theta}\left[2 x p_{2 n-1}(x)-p_{2 n-2}(x)-p_{2 n}(x)\right] \\
= & {\left[2 \frac{\gamma_{2 n+1}}{\gamma_{2 n+2}}-1\right] p_{2 n+2}(x)+2 \alpha_{2 n+1} p_{2 n+1}(x)+\left[2 \frac{\gamma_{2 n}}{\gamma_{2 n+1}}-1\right] p_{2 n}(x) } \\
& -e^{2 i \theta}\left\{\left[2 \frac{\gamma_{2 n-1}}{\gamma_{2 n}}-1\right] p_{2 n}(x)+2 \alpha_{2 n-1} p_{2 n-1}(x)\right. \\
& \left.+\left[2 \frac{\gamma_{2 n-2}}{\gamma_{2 n-1}}-1\right] p_{2 n-2}(x)\right\}
\end{aligned}
$$

Thus by (24)

$$
\lim _{n \rightarrow \infty}\left|2 x \psi_{2 n+1}(\theta)-\psi_{2 n}(\theta)-\psi_{2 n+2}(\theta)\right|=0
$$

uniformly inside $A$. Therefore (26) implies that

$$
\psi(\theta)=\lim _{n \rightarrow \infty} e^{(2 n+1) i \theta} \psi_{2 n+1}(\theta)
$$

is also true and the convergence is uniform inside $A$. Applying (16) repeatedly 
we obtain

$$
\left|\psi_{9}(\theta)\right|+\left|\psi_{10}(\theta)\right| \leqslant\left[\left|\psi_{n}(\theta)\right|+\left|\psi_{n+1}(\theta)\right|\right] \exp \left\{\frac{1}{|\sin 2 \theta|} \sum_{k=11}^{n+1} \delta_{k}\right\}
$$

where $n \geqslant 11$ is any odd integer. Therefore

$$
\left[\left|\psi_{9}(\theta)\right|+\left|\psi_{10}(\theta)\right|\right] \leqslant 2 \limsup _{n \rightarrow \infty}\left|\psi_{n}(\theta)\right| \cdot \exp \left\{\frac{1}{|\sin 2 \theta|} \sum_{k=11}^{\infty} \delta_{k}\right\} .
$$

We have

$$
|\sin 2 \theta|\left[\left|p_{9}(x)\right|+\left|p_{10}(x)\right|\right]<\left|\psi_{9}(\theta)\right|+\left|\psi_{10}(\theta)\right| .
$$

Since two consecutive orthogonal polynomials have no common zero we get

$$
\text { const } \cdot|\sin 2 \theta| \leqslant\left|\psi_{9}(\theta)\right|+\left|\psi_{10}(\theta)\right| \text {. }
$$

Thus by (26) and (27)

$$
|\psi(\theta)|^{-1} \leqslant \exp \{\text { const } /|\sin 2 \theta|\}
$$

for $x \in[-1,1]$.

Now we will show (22). From

$$
\left|\psi_{n}(\theta)\right|^{2}=p_{n}^{2}(x)-2 \cos 2 \theta p_{n}(x) p_{n-2}(x)+p_{n-2}^{2}(x)
$$

we obtain

$$
|\psi(\theta)|^{2}=\lim _{n \rightarrow \infty} \frac{1}{n} \sum_{k=1}^{n}\left[p_{k}^{2}(x)-2 \cos 2 \theta p_{k}(x) p_{k-2}(x)+p_{k-2}^{2}(x)\right] .
$$

It was shown in [3, Theorem 4.1.19] that if $l$ is a fixed nonnegative integer then

$$
\lim _{n \rightarrow \infty} \frac{\sum_{k=0}^{n} p_{k}(x) p_{k+l}(x)}{\sum_{k=0}^{n} p_{k}^{2}(x)}=\cos l \theta
$$

provided that $\alpha \in M, x \in[-1,1]$ and $\alpha$ is continuous at $x$. Thus

$$
\lim _{n \rightarrow \infty} \frac{\sum_{k=1}^{n}\left[p_{k}^{2}(x)-2 \cos 2 \theta p_{k}(x) p_{k-2}(x)+p_{k-2}^{2}(x)\right]}{\sum_{k=1}^{n} p_{k}^{2}(x)}=2\left(1-\cos ^{2} 2 \theta\right)
$$

for almost every $x \in[-1,1]$. Therefore

$$
|\psi(\theta)|^{2}=2 \sin ^{2} 2 \theta \lim _{n \rightarrow \infty} \frac{1}{n} \sum_{k=1}^{n} p_{k}^{2}(x),
$$

in particular,

$$
|\psi(\theta)|^{2}=2 \sin ^{2} 2 \theta \liminf _{n \rightarrow \infty} \frac{1}{n} \sum_{k=1}^{n} p_{k}^{2}(x)
$$

for almost every $x \in[-1,1]$. By Theorem 6.2.54 of [3] 


$$
\operatorname{iminf}_{n \rightarrow \infty} \frac{1}{n} \sum_{k=1}^{n} p_{k}^{2}(x)=\frac{1}{\pi \alpha^{\prime}(x) \sqrt{1-x^{2}}}
$$

for almost every $x \in[-1,1]$ whenever $\alpha \in M$. Consequently

$$
|\psi(\theta)|^{2}=\frac{8 x^{2} \sqrt{1-x^{2}}}{\pi \alpha^{\prime}(x)}
$$

for almost every $x \in[-1,1]$. Changing $\alpha^{\prime}$ on a set of measure zero we get (22) for every $x \in A$.

THEOREM 5. Let (10) be satisfied. Then da can be written in the form

$$
d \alpha(x)=w(x) d x+\Sigma\{\text { jumps outside }(-1,0) \cup(0,1)\}
$$

where $w$ is a continuous function on $(-1,0) \cup(0,1)$ and satisfies the inequality

$$
w(x)^{ \pm 1} \leqslant \exp \left\{\frac{\text { const }}{|x| \sqrt{1-x^{2}}}\right\}
$$

for $-1<x<1$. For the corresponding orthogonal polynomials the asymptotic formula

$$
\begin{array}{r}
\sqrt{w(x) \sqrt{1-x^{2}}} p_{n}(d \alpha, x)=\sqrt{\frac{2}{\pi}} \cos [n \theta+\Gamma(\theta)]+\sigma(1) \\
(x=\cos \theta)
\end{array}
$$

as $n \rightarrow \infty$ holds uniformly inside $(-1,0) \cup(0,1)$ where $\Gamma$ is a continuous function on $(-1,0) \cup(0,1)$.

Proof. The asymptotic formula (28) follows immediately from Lemma 4 since $\sin 2 \theta p_{n}=-\operatorname{Im} \psi_{n+2}$. Because $\psi$ is continuous and different from 0 for $x \in(-1,0) \cup(0,1)$ the argument of $\psi$ is also continuous for $x \in(-1,0) \cup$ $(0,1)$. Thus $\Gamma$ is a continuous function on $(-1,0) \cup(0,1)$. Now assume that $\alpha$ has a jump at some $x^{*} \in(-1,0) \cup(0,1)$. Then

$$
\sum_{n=1}^{\infty} p_{n}^{2}\left(x^{*}\right)<\infty
$$

has to be true. (See e.g. [3].) Thus

$$
\lim _{n \rightarrow \infty} p_{n}\left(x^{*}\right)=0
$$

must hold. Consequently $\psi\left(\theta^{*}\right)=0\left(x^{*}=\cos \theta^{*}\right)$ should be satisfied. But this contradicts (22). Therefore $\alpha$ has no jump in $(-1,0) \cup(0,1)$. By Theorem 3.3.7 of [3] the absolutely continuous and the singular components of $d \alpha$ are supported in $[-1,1]$ whenever $\alpha \in M$. Therefore the theorem will 
be proved if we show that

$$
\int_{\Delta} d \alpha_{s}=0
$$

for every interval $\Delta \subset(-1,1)$. We can assume without loss of generality that $0 \notin \Delta$. By Theorem 4.2.14 of [3] if $\alpha \in M$ then

$$
\lim _{n \rightarrow \infty} \int_{\Delta} p_{n}^{2} d \alpha=\frac{1}{\pi} \int_{\Delta} \frac{d x}{\sqrt{1-x^{2}}} .
$$

We obtain from the asymptotic formula (28) that

$$
\lim _{n \rightarrow \infty} \frac{1}{n} \sum_{k=1}^{n} p_{k}^{2}(x)=\frac{1}{\pi \alpha^{\prime}(x) \sqrt{1-x^{2}}}
$$

uniformly for $x \in \Delta$. Therefore

$$
\begin{array}{r}
\lim _{n \rightarrow \infty} \frac{1}{n} \sum_{k=1}^{n} \int_{\Delta} p_{k}^{2}(x) d \alpha(x)=\frac{1}{\pi} \int_{\Delta} \frac{1}{\alpha^{\prime}(x) \sqrt{1-x^{2}}} d \alpha(x) \\
=\frac{1}{\pi} \int_{\Delta} \frac{d x}{\sqrt{1-x^{2}}}+\frac{1}{\pi} \int_{\Delta} \frac{1}{\alpha^{\prime}(x) \sqrt{1-x^{2}}} d \alpha_{s}(x)
\end{array}
$$

Using (30) and (32) we get

$$
\int_{\Delta} \frac{1}{\alpha^{\prime}(x) \sqrt{1-x^{2}}} d \alpha_{s}(x)=0
$$

which implies (29) since $\alpha^{\prime}(x) \sqrt{1-x^{2}}$ is uniformly bounded for $x \in \Delta$.

THEOREM 6. If (9) holds then $\log \alpha^{\prime}(\cos \theta)$ is integrable on $[0,2 \pi]$.

Proof. Since $\sin \theta \alpha^{\prime}(\cos \theta)$ is integrable the function $\log ^{+} \alpha^{\prime}(\cos \theta)$ is certainly integrable. Therefore we have to show that

$$
\int_{0}^{2 \pi} \log ^{+} \frac{1}{\alpha^{\prime}(\cos \theta)} d \theta<\infty
$$

From (9) follows that

$$
\sum_{j=4}^{\infty} \log j \delta_{j}<\infty
$$

where the numbers $\delta_{j}$ were defined in Lemma 1 . Let $n=5,6, \ldots$ the sets $e_{n}$ be defined by

$$
e_{n}=\bigcup_{k=0}^{3}\left[\frac{k \pi}{2}-\frac{1}{10 n^{2}}, \frac{k \pi}{2}+\frac{1}{10 n^{2}}\right]
$$


Then $e_{n} \subset e_{n-1}$ and $\left|e_{n-2} \backslash e_{n}\right|<32(n-2)^{-3}$. We have by (15)

$$
\log ^{+}\left[\left|\psi_{n}(\theta)\right|+\left|\psi_{n-1}(\theta)\right|\right]<\log ^{+}\left[\left|\psi_{n-2}(\theta)\right|+\left|\psi_{n-3}(\theta)\right|\right]+\frac{\delta_{n}+\delta_{n-1}}{|\sin 2 \theta|}
$$

for $n=10,11, \ldots$ Therefore

$$
\begin{aligned}
\int_{[0,2 \pi] \backslash e_{n}} \log ^{+} & {\left[\left|\psi_{n}(\theta)\right|+\left|\psi_{n-1}(\theta)\right|\right] d \theta } \\
< & \int_{[0,2 \pi] \backslash e_{n-2}} \log ^{+}\left[\left|\psi_{n-2}(\theta)\right|+\left|\psi_{n-3}(\theta)\right|\right] d \theta \\
& +\left|e_{n-2} \backslash e_{n}\right| \max \log ^{+}\left[\left|\psi_{n-2}\right|+\left|\psi_{n-3}\right|\right] \\
& +\left(\delta_{n}+\delta_{n-1}\right) \int_{[0,2 \pi] \backslash e_{n}} \frac{d \theta}{|\sin 2 \theta|} .
\end{aligned}
$$

By (18) and (34)

$$
\max \log ^{+}\left[\left|\psi_{n-2}\right|+\left|\psi_{n-3}\right|\right]<\operatorname{const}(n-2) .
$$

By the construction of $e_{n}$

$$
\int_{[0,2 \pi] \backslash e_{n}} \frac{d \theta}{|\sin 2 \theta|}<\text { const } \log (n-1) .
$$

Hence we obtain

$$
\begin{aligned}
\int_{[0,2 \pi] \backslash e_{n}} \log ^{+}\left[\left|\psi_{n}(\theta)\right|+\left|\psi_{n-1}(\theta)\right|\right] d \theta \\
\quad<\int_{[0,2 \pi] \backslash e_{n-2}} \log ^{+}\left[\left|\psi_{n-2}(\theta)\right|+\left|\psi_{n-3}(\theta)\right|\right] d \theta \\
\quad+\operatorname{const}\left[\frac{1}{(n-2)^{2}}+\delta_{n-1} \log (n-1)+\delta_{n} \log n\right]
\end{aligned}
$$

Repeated application of this inequality gives us

$$
\begin{aligned}
\int_{[0,2 \pi] \backslash e_{n}} \log ^{+}\left[\left|\psi_{n}(\theta)\right|+\left|\psi_{n-1}(\theta)\right|\right] d \theta< & \int_{[0,2 \pi] \backslash e_{10}} \log ^{+}\left[\left|\psi_{10}(\theta)\right|+\left|\psi_{9}(\theta)\right|\right] d \theta \\
& + \text { const }\left[\sum_{k=10}^{n-2} \frac{1}{k^{2}}+\sum_{k=10}^{n} \log k \delta_{k}\right]
\end{aligned}
$$

Recall that $\left|e_{n}\right| \rightarrow 0$ when $n \rightarrow \infty$. Thus by (34)

$$
\int_{0}^{2 \pi} \liminf _{n \rightarrow \infty} \log ^{+}\left[\left|\psi_{n}(\theta)\right|+\left|\psi_{n-1}(\theta)\right|\right] d \theta<\infty .
$$

Consequently (33) follows from Lemma 4. 
THEOREM 7. If (8) is satisfied then there exist three positive numbers $a, b$ and c such that

$$
p_{n}^{2}(d \alpha, x)<c|x|^{-a}\left(1-x^{2}\right)^{-b} \quad(-1<x<1)
$$

for $n=1,2, \ldots$ and

$$
\alpha^{\prime}(x)>c^{-1}|x|^{a}\left(1-x^{2}\right)^{b} \quad(-1<x<1) .
$$

Proof. It follows from (8) that

$$
\sum_{j=4}^{n} j \delta_{j}<\text { const } \log n
$$

where $\delta_{j}$ were defined in Lemma 1 . Therefore by (18)

$$
\left|\psi_{n}(\theta)\right|<n^{c_{4}}
$$

$n=10,11, \ldots$ and $-1<x<1$ with some fixed constant $c_{4}$. Since $\left|\sin 2 \theta p_{\mathrm{n}}\right|=\left|\operatorname{Im} e^{-2 i \theta} \psi_{n}\right|$ inequality (35) will hold if we can show that from the estimate

$$
|\sin 2 \theta|^{c_{s}}\left|\psi_{n}(\theta)\right|<\text { const } n^{c_{6}}
$$

$(n=10,11, \ldots$ and $-1<x<1)$ the inequality

$$
|\sin 2 \theta|^{c_{5}+1}\left|\psi_{n}(\theta)\right|< \begin{cases}\text { const } & \text { if } 0<c_{6}<1, \\ \text { const } n^{c_{6}-1 / 2} & \text { if } c_{6}>1\end{cases}
$$

( $n=10,11, \ldots$ and $-1<x<1$ ), follows whenever (37) is satisfied. We have

$$
\left|\psi_{n}(\theta)\right|<\sum_{k=10}^{n}\left|\psi_{k}(\theta)-e^{-2 i \theta} \psi_{k-2}(\theta)\right|+\left|\psi_{8}(\theta)\right|+\left|\psi_{9}(\theta)\right| .
$$

Thus by Lemma 1

$$
\left|\psi_{n}(\theta)\right|<\sum_{k=10}^{n} \delta_{k}\left[\left|p_{k-2}(x)\right|+\left|p_{k-3}(x)\right|\right]+\text { const. }
$$

Therefore we obtain

$$
\left|\sin 2 \theta \psi_{n}(\theta)\right|<\sum_{k=10}^{n} \delta_{k}\left[\left|\psi_{k-2}(\theta)\right|+\left|\psi_{k-3}(\theta)\right|\right]+\text { const. }
$$

Using (38) we get

$$
|\sin 2 \theta|^{c_{s+1}}\left|\psi_{n}(\theta)\right|<\operatorname{const}\left[\sum_{k=10}^{n} k^{c_{6}} \delta_{k}+1\right] .
$$

If $0<c_{6}<1$ then by (37)

$$
\sum_{k=10}^{\infty} k^{c} \delta_{k}<\infty
$$


and (39) follows. Otherwise

$$
\sum_{k=10}^{n} k^{c_{8} \delta_{k}}<n^{c_{6}-1 / 2} \sum_{k=10}^{n} \sqrt{k} \delta_{k} .
$$

Since by (37)

$$
\sum_{k=10}^{\infty} \sqrt{k} \delta_{k}<\infty
$$

inequality (39) follows again. Thus we have proved (35). The estimate (36) for $\alpha^{\prime}$ follows immediately from (35) and (31).

\section{REFERENCES}

1. K. M. Case, Orthogonal polynomials revisited, Theory and Application of Special Functions, (R. A. Askey, ed.), Academic Press, New York, 1975, pp. 289-304.

2. J. Favard, Sur les polynomes de Tchebicheff, C. R. Acad. Sci. Paris 200 (1935), 2052-2053.

3. P. G. Nevai, Orthogonal polynomials, Mem. Amer. Math. Soc. (to appear).

4. __ On orthogonal polynomials, J. Approximation Theory (to appear).

5. __ Distribution of zeros of orthogonal polynomials, Trans. Amer. Math. Soc. (to appear).

6. G. Szegö, Orthogonal polynomials, 3rd ed., Amer. Math. Soc. Colloq. Publ., vol. 23, Amer. Math. Soc., Providence, R.I., 1967.

Department of Mathematics, Ohio State University, Columbus, Oho 43210 